Interfaces

\title{
Événement historique, événement médiatique : exposer l'attentat. Étude des usages multimédias dans le National 9/11 Memorial Museum
}

\section{Claire Ducresson-Boët}

\section{(2) OpenEdition}

\section{Journals}

Édition électronique

URL : http://journals.openedition.org/interfaces/650

DOI : 10.4000/interfaces.650

ISSN : 2647-6754

Éditeur :

Université de Bourgogne, Université de Paris, College of the Holy Cross

\section{Édition imprimée}

Date de publication : 21 juin 2019

Pagination : 117-136

ISSN : $1164-6225$

\section{Référence électronique}

Claire Ducresson-Boët, «Événement historique, événement médiatique : exposer l'attentat. Étude des usages multimédias dans le National 9/11 Memorial Museum », Interfaces [En ligne], 41 | 2019, mis en ligne le 21 juin 2019, consulté le 07 janvier 2021. URL : http://journals.openedition.org/interfaces/650 ; DOI : https://doi.org/10.4000/interfaces.650

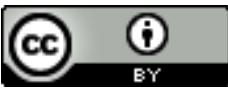

Les contenus de la revue Interfaces sont mis à disposition selon les termes de la Licence Creative Commons Attribution 4.0 International. 


\title{
ÉVÉNEMENT HISTORIQUE, ÉVÉNEMENT MÉDIATIQUE : EXPOSER L'ATTENTAT. ÉTUDE DES USAGES MULTIMÉDIAS DANS LE NATIONAL 9/11 MEMORIAL MUSEUM
}

\author{
Claire Ducresson-Boët \\ Université Paris Diderot 7 (LARCA), Université du Québec (Montréal)
}

Le 15 mai 2014, un nouveau musée est inauguré à Manhattan, New York. Après huit ans de construction, des centaines de millions de dollars et de nombreux différends, le National September 11 Memorial Museum (ou 9/11 Museum dans son appellation abrégée) ouvre enfin ses portes sous ce qui fut jadis les tours jumelles du World Trade Center. Dédié aux 2977 victimes des quatre attentats terroristes qui frappèrent les États-Unis le 11 septembre 2001, ainsi qu'aux six victimes de celle déjà perpétrée le 26 février 1993 contre le World Trade Center, il complète un monument commémoratif inauguré trois ans plus tôt sur le même site et sous lequel il est enterré, le National September 11 Memorial (figure 1). Conçu par l'agence d'architecture Davis Brody Bond, le musée occupe 10000 mètres carrés, à vingt et un mètres de profondeur ${ }^{1}$, et possède dans ses collections plus de 10000 artefacts sauvés des attentats. Objets personnels des victimes, vestiges des tours jumelles ou débris d'avion, ils accompagnent 23000 images et près de 2000 témoignages oraux, répartis dans deux principales galeries, chacune située sous une ancienne tour, et imaginées par les agences Thinc Design et Local Projects ; sous la tour sud se trouve l'exposition mémorielle In Memoriam, et sous la tour nord, l'exposition historique September 11 2001, Historical Exhibition (figure 2).

L'entrée dans le musée se fait par un pavillon dessiné par l'agence Snøhetta, situé à la surface de Ground Zero, entre les deux bassins du mémorial. 


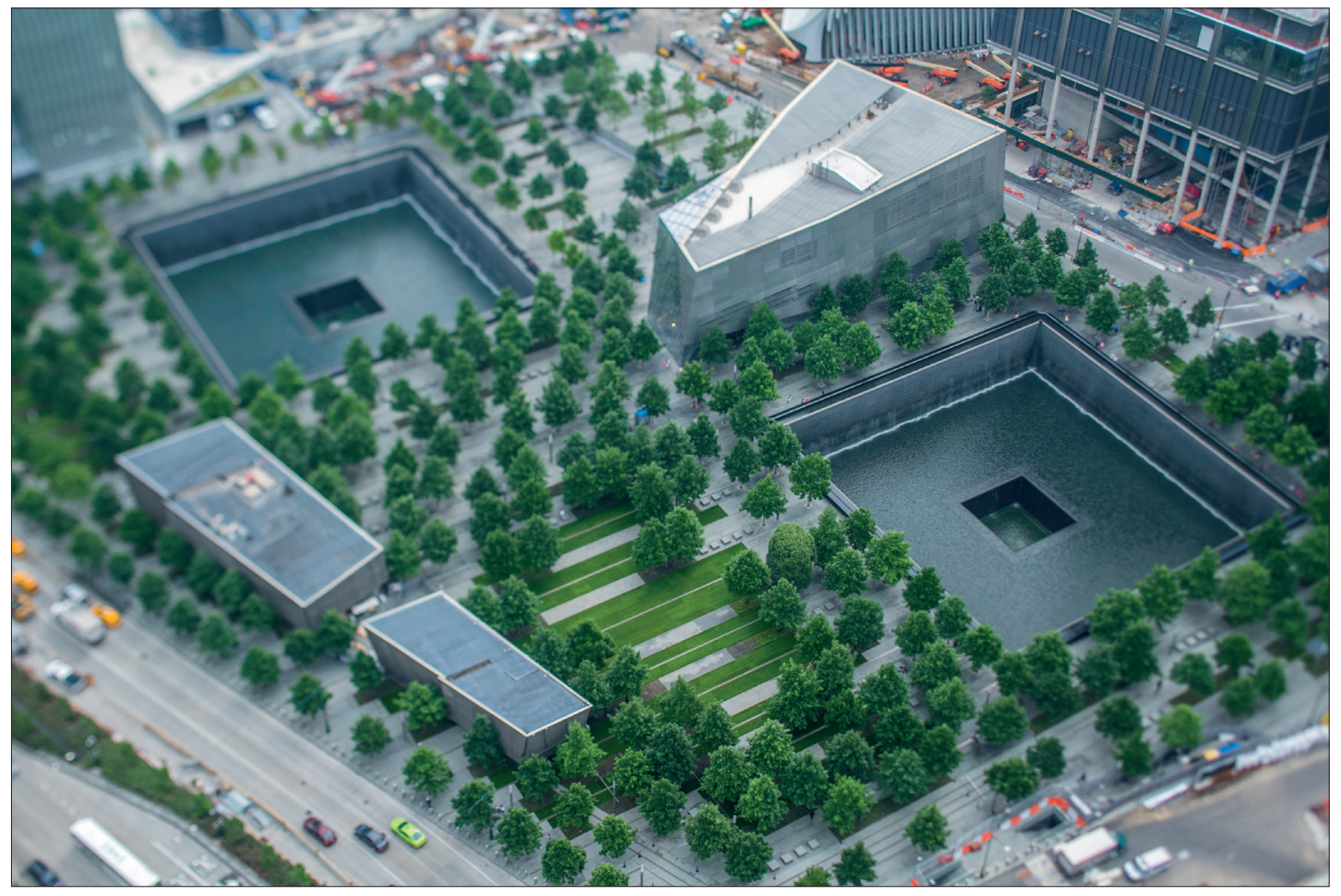

Figure 1 : Vue aérienne du National 9/11 Memorial and Museum. Entre les deux bassins, on peut voir le pavillon d'entrée du National 9/11 Memorial Museum. Courtesy 9/11 Memorial Museum. Photographie de Jin S. Lee. 


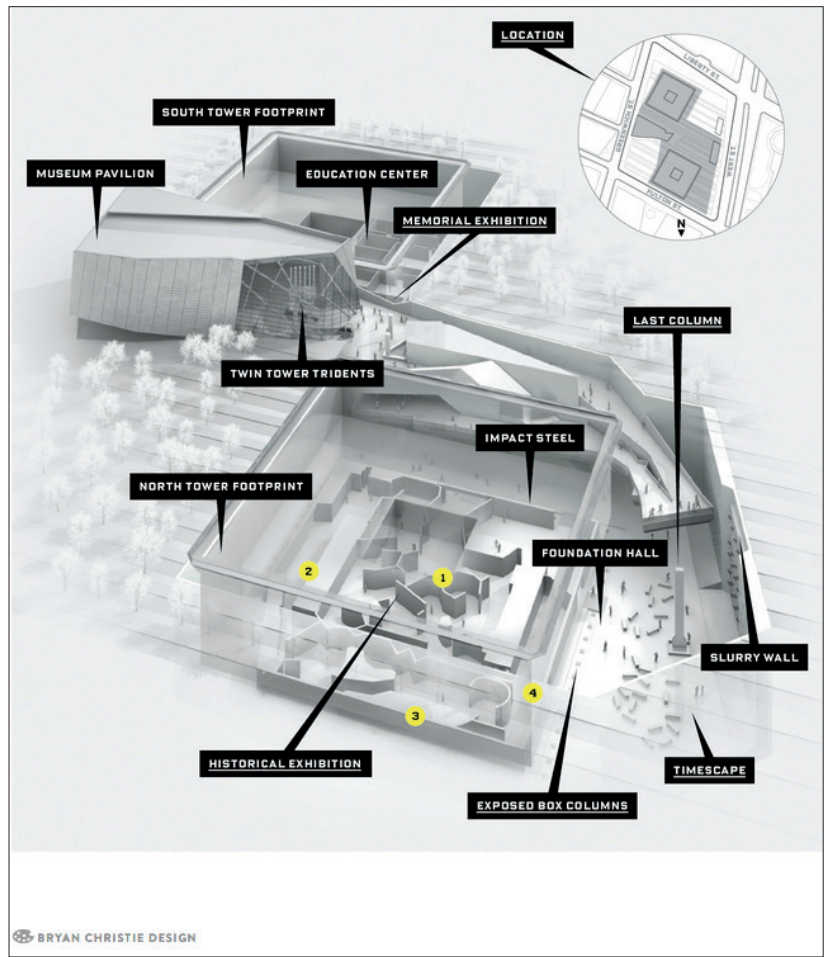

Figure 2 : Rendu montrant l'organisation du musée sous le 9/11 Memorial. Courtesy Bryan Christie Design et Wired/Condé Nast.

Officiellement présenté comme la principale institution américaine vouée à l'étude des implications, conséquences et significations de ces attentats terroristes ${ }^{2}$, le National September 11 Memorial Museum semble de prime abord fidèle à la définition traditionnelle d'un musée - c'est-à-dire un établissement ouvert au public dans lequel sont collectés, préservés, interprétés et exposés des objets

2. Le site officiel du 9/11 Memorial and Museum le décrit comme : « the country’s principal institution concerned with exploring the implications of the events of 9/11, documenting the impact of those events and exploring 9/11's continuing significance ». Voir https://www.911memorial.org/museum (page consultée le 20 mai 2019). 
d'intérêt artistique, anthropologique, historique, technique ou scientifique à des fins de développement et de diffusion des connaissances (Pearce 30 ; Goode 113). Toutefois, son emplacement géographique, sa genèse et les débats menés tout au long de sa réalisation en font une institution à la croisée du musée et du mémorial et dans laquelle affect, identité, deuil et patriotisme imprègnent le discours mis en scène. Les attentats du 11 septembre 2001, et notamment les attaques contre les tours jumelles, furent un événement médiatique sans précédent. Pensé comme un acte de communication par les terroristes et médiatisé en direct à travers le monde grâce à des technologies numériques alors en plein essor, il se déroula sous les yeux de près de deux milliards d'individus en temps réel et accompagne un tournant dans l'histoire des outils d'information et de communication. Ce rapport dialectique profond entre 11-Septembre et médias est reproposé tout au long du musée qui se distingue par la profusion des outils numériques qui y sont déployés, semble-t-il, dans une tentative de combler cet écart entre des ambitions à la fois pédagogiques et commémoratives. Alors que la visite s'ouvre sur la force médiatique des événements du 11 septembre 2001 et le rôle des médias de masse dans leurs captation et monstration - télévision et presse écrite principalement -, de nombreux objets numériques jalonnent ensuite le parcours du visiteur. Tantôt installations, tantôt supports pour des collections dématérialisées, ces technologies apparaissent comme de véritables recours narratifs pour mettre en espace un récit des plus sensibles à travers la parole du témoin notamment. Invité à une expérience multimédia interactive et immersive, le visiteur devient - ou redevient - à son tour témoin des attentats du 11 septembre 2001 .

Dédié à une série d'événements extrêmement médiatisés, le 9/11 Museum fait la part belle aux médias dans ses expositions, se construisant par là même à l'image de son sujet, tout à la fois médiatique, spectaculaire et dramatique. En effet, avant de savoir si elle constituerait un événement historique, la destruction des tours jumelles du World Trade Center dans la matinée du 11 septembre 2001 fut d'abord un événement médiatique. L'une des cibles des attentats qui frappèrent également le Pentagone, le World Trade Center fut choisi non seulement pour le symbole de la toute-puissance américaine qu'il représentait, mais également pour la gémellité de ses deux tours, les plus hautes de New York et du monde. Comme le souligne Clément Chéroux dans l'ouvrage qu'il consacre à la représentation visuelle de l'événement dans les médias, les actes de terrorisme sont des actes de communication dont le langage est celui de la barbarie et dont les médias de masse sont un des moyens de mise en œuvre (12) - plus de quinze ans après le 11-Septembre, cela demeure manifeste dans le fonctionnement d'une organisation telle que l'État Islamique. L'efficacité du terrorisme, écrit Chéroux, « repose [...], pour une bonne part, sur sa compréhension, puis son exploitation, du système médiatique » (12). La redondance des tours jumelles de New York fut ainsi un élément primordial dans la conception de l'attentat new-yorkais. Il ne s'agissait pas seulement de détruire un symbole, il fallait en plus le détruire sous les yeux du plus grand nombre, et les terroristes n'étaient pas sans 
savoir qu'après que le premier avion eut frappé la tour nord, tous les regards se tourneraient vers les tours jumelles et assisteraient alors à l'impact du second avion. L'attentat de New York fut donc conçu « pour la médiatisation et par la répétition » (16).

De fait, le double attentat perpétré contre les tours jumelles du World Trade Center fut rapidement considéré comme l'événement le plus photographié, non seulement de l'histoire du photojournalisme (35), mais également de celle de la photographie (Kirshenblatt-Gimblett 12). L'attentat fut capté par des photographes et photojournalistes professionnels bien sûr, mais également, fait alors nouveau, par un grand nombre de photographes amateurs. Il fut donc ultra-médiatisé et images fixes et mobiles inondèrent les grands médias, écrits et télévisuels, américains comme étrangers. Pour la première fois, les images de l'événement firent partie intégrante de l'événement lui-même (20). Le 11-Septembre marque ainsi l'entrée fracassante du photojournalisme dans le XXI ${ }^{\text {ème }}$ siècle et incarne le profond bouleversement de notre rapport à l'événement engendré notamment par des technologies numériques alors nouvelles et en plein essor, au rang desquelles la photographie numérique et l'internet. Leur développement, alors que le champ du photojournalisme, en perpétuelle crise, se fragilise, et l'usage qui en est fait dès 2001 ont modifié durablement la production et la diffusion de l'information dans les sociétés occidentales. Aussi est-il intéressant de constater la présence prégnante de nombreux médias dans le National September 11 Memorial Museum voué à la muséification et à la commémoration du 11 septembre 2001, faisant de lui une véritable institution multimédia.

Les médias de masse occupent une place de choix dans le musée et ouvrent le parcours du visiteur. On peut ainsi lire dans le texte inaugural de la visite qu' « environ deux milliards de personnes, soit près d'un tiers de la population mondiale, auraient été témoins le jour même de ces horribles événements en personne ou via les retransmissions télévisuelles, radiophoniques ou sur l'internet $»^{3}$. En effet, l'instantanéité rendue possible par les nouvelles technologies d'alors a fait du 11-Septembre un événement diffusé en direct sur la planète, ce que Jean-François Sirinelli appelle « un événementmonde » : «La concomitance entre l'attentat et son onde de choc quasi instantanée lui a bien conféré un tel statut : l'événement-monstre a été, en même temps, un événement-monde. Guernica, en quelque sorte, en direct sur CNN ! » (35). C'est dans l'exposition dite « historique » que les médias de masse traditionnels - télévision et presse écrite - sont le plus présents, notamment à travers les « icônes » des événements. On retrouve ainsi la célèbre photographie de Thomas Franklin des trois pompiers hissant le drapeau américain sur les ruines des tours jumelles, le Falling Man de Richard Drew pour Associated Press, ou encore Dust Lady de Stan Honda pour l'Agence France-Presse. Des écrans diffusent

Je traduis : « Approximately two billion people, almost one third of the world population, are estimated to have witnessed these horrific events directly or via television, radio and internet broadcasts that day ». 
également en boucle des passages de journaux télévisés montrant le crash du second avion dans la tour sud. Enfin, de nombreuses unes de presse du monde entier sont reproduites et accrochées aux cimaises dans un effet d'abondance et de redondance classique dans l'exposition du 11-Septembre puisque l'on retrouve un accrochage fort semblable dans la 9/11 Gallery du Newseum de Washington, D.C. Dans l'enceinte du musée, se répète ainsi le phénomène de diplopie finement analysé par Clément Chéroux, celui d'une « répétitivité iconique » (17) malgré une profusion d'images.

À travers cette place accordée aux médias de masse apparaît une première figure du témoin, celle du journaliste. Témoin de profession, à la parole neutre et globalisante mise au service du bien commun, le journaliste est un personnage de premier plan dans le récit du 11-Septembre. Au même titre que le pompier ou le secouriste, il est devenu l'un des héros de cette journée, prêt à donner sa vie, non pas pour sauver des victimes, mais pour raconter l'événement. Si les attentats furent vécus comme un acte de guerre, l'appréciation du travail des journalistes ce jour-là fut donc, sans grande surprise, semblable à celle d'un reporter de guerre, " "chevalier des temps modernes" qui met sa vie en danger pour la cause journalistique » (Gervais 12). Cette célébration du journaliste dans le musée témoigne de la relation ancienne et « symbiotique entre les médias américains et la nation américaine », au prisme de laquelle il faut examiner " l'émotion patriotique » produite par les attentats du 11 septembre 2001 (Frau-Meigs 155). À travers ce lien « symbiotique » et caractéristique des États-Unis, les journalistes apparaissent en effet comme les garants de la liberté d'expression, un des piliers de l'idéal américain figurant au premier amendement de la Constitution. Dans l'ouvrage Running Toward Danger: Stories Behind the Breaking News of 9/11 publié par le Newseum ${ }^{4}$ en 2002, Rod Dreher, journaliste au New York Post, écrit ainsi : « Il y a trois catégories de personnes qui courent vers une catastrophe plutôt que de la fuir : les policiers, les pompiers et les journalistes $\rangle^{5}(34)$. Là où le $9 / 11$ Museum innove quant à l'intégration des médias de masse à son récit, c'est avec Timescape (figure 3), une visualisation de données (data visualization), vidéo développée par Local Projects et projetée à la fin du parcours.

4. Le Newseum situé à Washington, D.C., à quelques encablures des plus hauts symboles du pouvoir fédéral (la Maison Blanche, le Capitole, la Cour Suprême entre autres) ou du patrimoine national (les musées de la Smithsonian et les monuments commémoratifs du National Mall notamment) est lui-même symptomatique de cette place des médias dans la nation américaine. Cet immense musée, fondé par l'homme d'affaire et créateur de USA Today, Al Neuharth, et financé par une fondation issue de Gannet News, est à la gloire du journalisme. Avec un coût estimé de 450 millions de dollars, il est l'un des musées les plus chers construits aux États-Unis et compte parmi ses soutiens financiers News Corp., la famille Hearst, Disney, Time Warner, ou encore NBC Universal.

Je traduis : " There are three kinds of people who run toward the disaster, not away: cops, firemen and reporters » (Newseum 2002). 


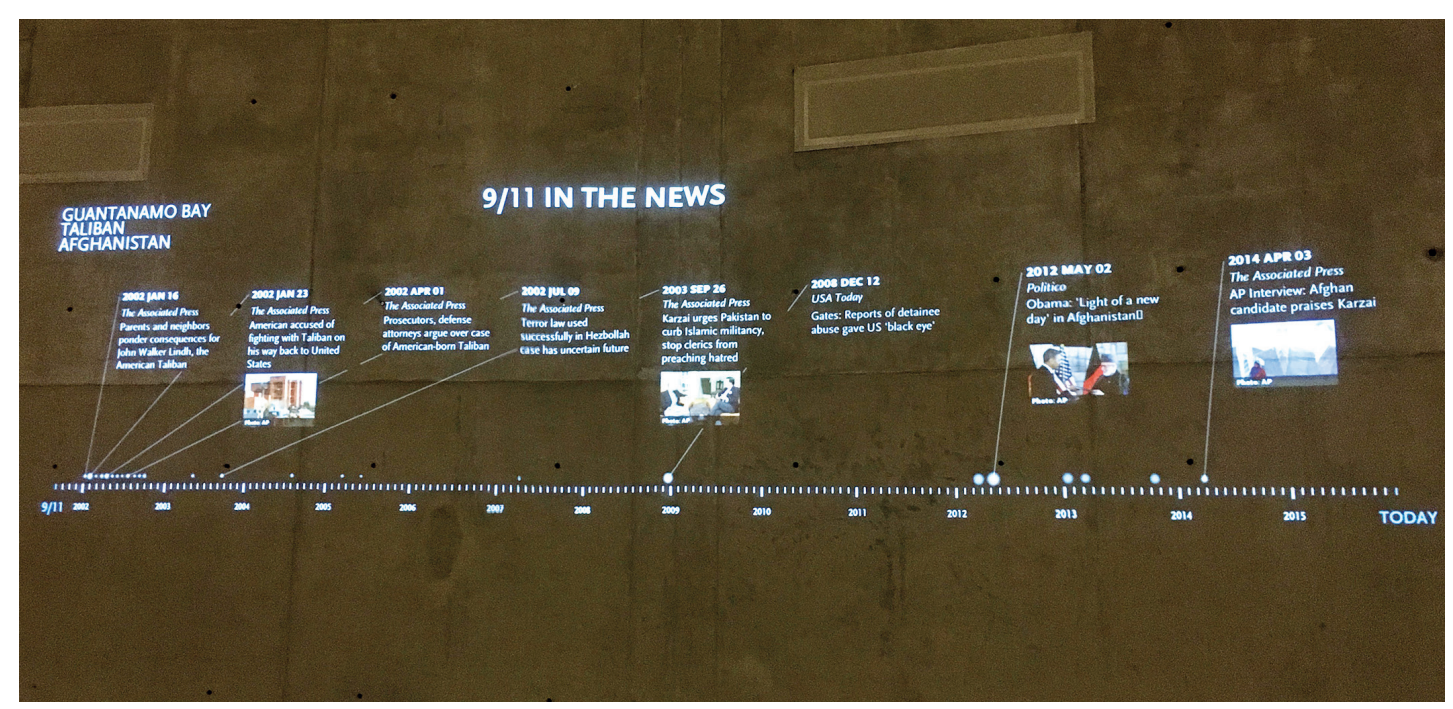

Figure 3 : Vue de Timelapse. Les résultats sont ici générés d'après les trois mots-clefs « Guantanamo Bay », « Taliban » et « Afghanistan ». Photographie de Claire Ducresson-Boët.

L'installation repose sur une base de données quotidiennement mise jour et recensant désormais quelque quatre millions d'articles de presse datés du 11 septembre 2001 à aujourd'hui ; un algorithme sélectionne de façon aléatoire trois mots ou expressions clefs associés au 11-Septembre et ratisse cette base à la recherche des articles où figurent ces trois termes ${ }^{6}$. Les résultats sont organisés le long d'une frise chronologique allant du 11 septembre 2001 à aujourd'hui, faisant figurer la date, la source et le titre des articles trouvés, et parfois une photographie les illustrant ${ }^{7}$. Comme il est stipulé dans le texte qui la présente, cette installation a pour but d'attester de la portée et de la persistance de l'onde de choc du 11-Septembre. Selon Lauren F. Klein, le recours à une installation vidéo générée par un algorithme traduit un désir d'objectivité dans le commissariat du musée, dont elle cherche à prendre les apparences (435).

6. Ces groupes de mots peuvent être par exemple « crashed, flight, Shanksville », « Afghanistan, Pakistan, Osama Bin Laden », « Rudolph Giuliani, George Pataki, New York », ou encore « Guantanamo Bay, Taliban, Afghanistan ».

7. Pour une description détaillée de Timescape, voir Lauren F. Klein. 
Par ces différents recours à des médias de masse nationaux et internationaux, les attentats du 11-Septembre sont présentés comme un événement dont les enjeux et implications dépassent largement les frontières américaines. À travers la figure du journaliste-témoin, le 11-Septembre est raconté comme une expérience « globale plutôt que locale qui a unifié, bien que pour un court instant, une vision du monde » (Sturken, « The 9/11 Memorial Museum » 479) ${ }^{8}$. Il apparait ainsi comme l'affaire de tous et non plus comme le résultat, certes dramatique, de la politique étrangère menée par les États-Unis depuis des décennies. Comme le souligne Erika Doss dans Memorial Mania: Public feeling in America, la contextualisation du 11-Septembre dans la politique étrangère américaine ou dans l'histoire du terrorisme aux États-Unis est très mal perçue dans l'opinion et souvent jugée comme un affront fait aux victimes $(122)^{9}$. L'appellation la plus répandue dans le langage courant pour désigner cette série d'attentats - à savoir « 9/11 » en anglais et «11-Septembre » en français - est, elle aussi, symptomatique de la décontextualisation à l'œuvre dans le récit de ces événements, dépouillés de leur année et de leur localisation ${ }^{10}$. Ils apparaissent alors comme quelque chose d'atemporel et apolitique. La mise en place des récits dans le musée entretient ainsi le mythe de l'exceptionnalisme de ces attentats, inattendus, imprévisibles, inexplicables.

Au-delà du journaliste ou du photojournaliste, le 9/11 Museum décline la figure du témoin tout au long de ces expositions, dans une sorte de subjectivation de l'événement. Le « témoin de circonstances ", passant qui se trouvait dans Lower Manhattan au moment des crashs, et le « témoin indirect » assistant pourtant presque en simultané à l'événement, souvent à des milliers de kilomètres de New York, sont représentés dès le début du parcours dans deux installations multimédias : We Remember et Witness. À quelques pas du texte inaugural précédemment cité, We Remember (figure 4) met en scène des témoignages issus des collections du musée.

8. Je traduis : « $[\ldots]$ we see the event as a global rather than local experience, and as one that unified, however briefly, a worldview ».

9. Erika Doss écrit : « attempts to contextualize 9/11 within narratives of either domestic or international terrorism, or within the nation's larger history of violence, are deemed disrespectful to the memory of those who died, and traitorous to the national duty of patriotic revenge » (Doss 122).

10. Une localisation pourtant essentielle, comme on l'a montré plus haut. En outre, le nombre 11 est régulièrement représenté de sorte à évoquer graphiquement les tours jumelles ; c'est notamment le cas du le logo du 9/11 Memorial Museum. Précisons à ce propos, que le 9/11 est souvent réduit à l'attaque contre les tours jumelles, d'une part du fait du nombre de personnes qui y périrent et de la symbolique de leur destruction, et d'autre part, peut-être, du fait de cette redondance visuelle entre les Twin Towers et la date des attentats. 


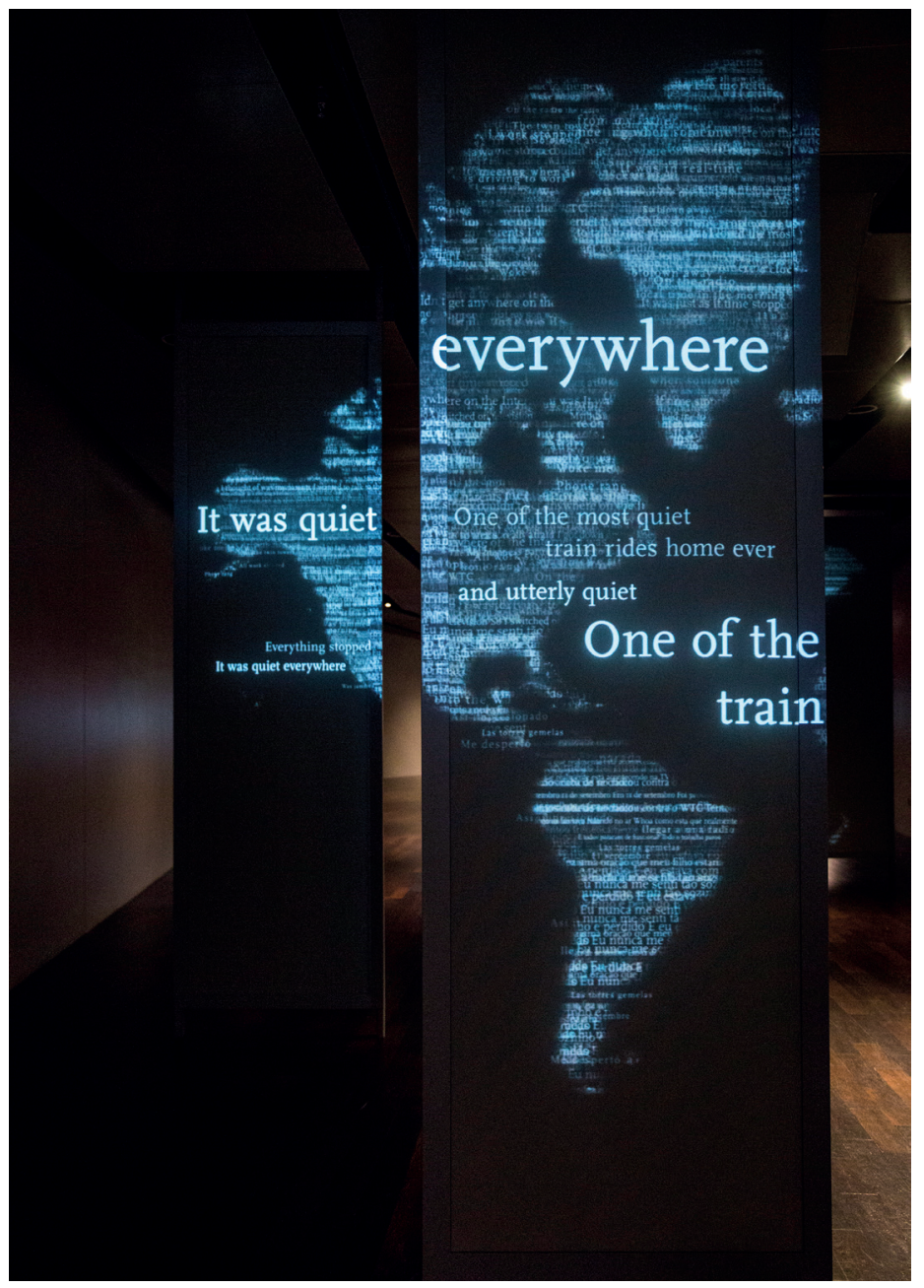

Figure 4 : Vue de l'installation We Remember.

Courtesy 9/11 Memorial Museum. Photographie de Jin S. Lee. 
Il s'agit d'une série de panneaux verticaux, installés en quinconces et sur lesquels sont projetées les transcriptions de témoignages d'individus à travers le monde se remémorant les circonstances dans lesquelles ils apprirent la nouvelle des attentats. Alors que les mots se succèdent sur les panneaux, les enregistrements vocaux correspondants sont également diffusés et lorsque le visiteur se place à un certain angle et à une certaine distance des panneaux, les textes projetés sur ceux-ci forment une carte du monde. We Remember met en scène ce que les psychologues Rober Brown et James Kulick ont identifié comme des « souvenirs flashs » (flashbulb memories) et que Barbara Kirshenblatt-Gimblett décrit comme le souvenir vif, si ce n'est précis, des circonstances dans lesquelles un individu a eu connaissance d'un événement public de grande importance, associant surprise, émotion intense et répercussions majeures (16). Cette première installation est immédiatement suivie de Witness (figure 5), une seconde série de panneaux sur lesquels défilent des photographies de témoins oculaires des attaques contre le World Trade Center, les yeux levés vers le ciel, l'air abasourdi ou horrifié ${ }^{11}$.

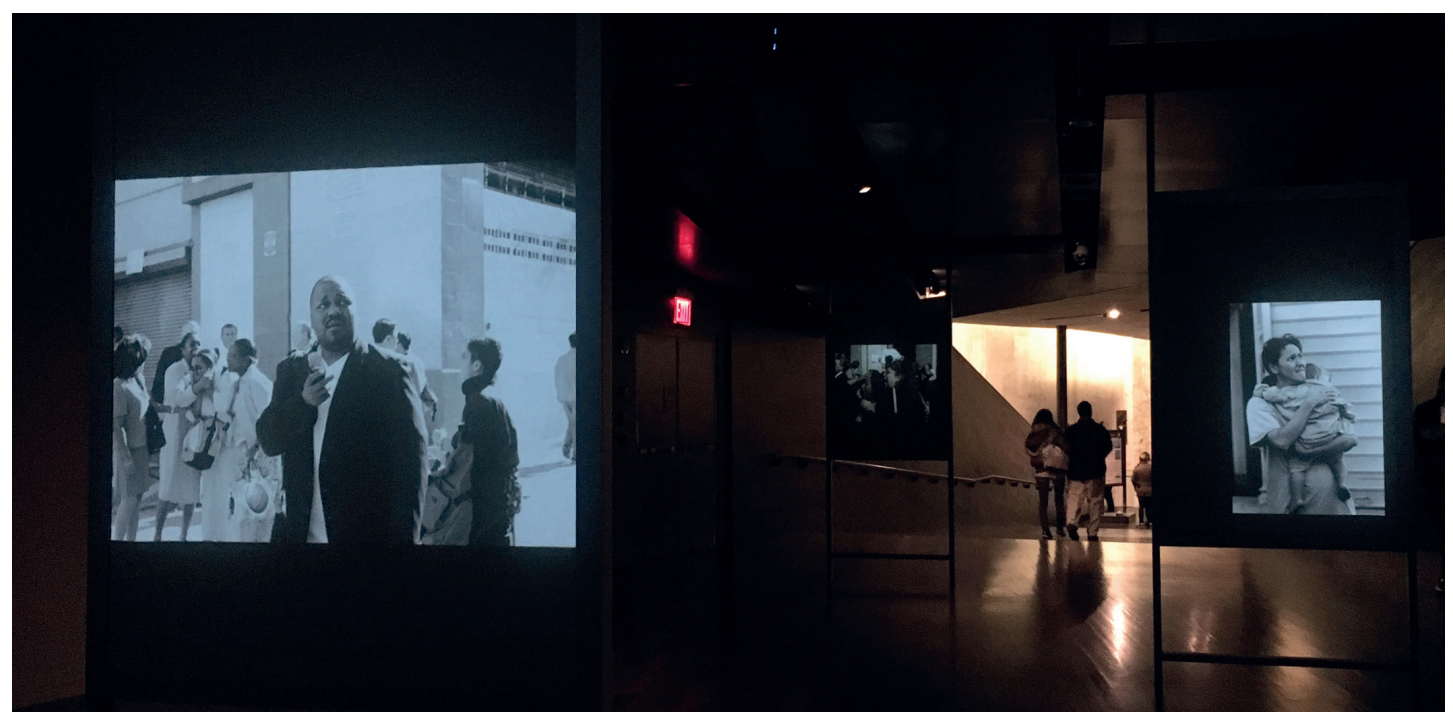

Figure 5 : Vue de l'installation Witness. Photographie de Claire Ducresson-Boët.

11. Ces photographies sont issues de l'exposition Here Is New York; plusieurs d'entre elles appartenant à la New York Historical Society, ont fait l'objet de dons au National 9/II Memorial Museum. 
L'objectif de l'appareil photo est alors braqué sur eux dans un cadrage resserré laissant les tours ou toute autre trace des attaques hors champ. À cette étape liminaire du parcours, le visiteur n'a donc pas encore vu les tours en feu; il n'a de l'attentat que des images, pour ainsi, dire en négatif. Ce qui nous est donné à voir, ce n'est pas l'attentat, mais sa perception et sa remémoration. Le 11-Septembre est ainsi d'emblée posé comme un événement global, stupéfiant et mémorable. Aussi les premiers protagonistes sont-ils les témoins, directs et indirects, professionnels et amateurs, de l'événement, au rang desquels le visiteur est invité à se placer à peine a-t-il commencé sa visite - il $\mathrm{y}$ a fort à parier que de nombreux visiteurs se prêtent à leur tour à l'exercice de remémoration mis en scène dans Remember. Cette figure, qui sera largement reprise dans la suite des expositions, s'ajoute ainsi à la tension dialectique entre 11-Septembre et médias. On l'a vu, la question de la répétition est au cœur de la conception même des attentats de New York; le crash de 8h46 dans la Tour Nord se répète à $9 \mathrm{~h} 03$ dans la Tour Sud; dans les heures puis les jours qui suivent les attentats, ceux-ci se répètent inlassablement dans les médias nationaux et internationaux diffusant en boucle et publiant à l'envi les images des crashs, des tours en feu puis de leur effondrement. C'est dans cette économie de la répétition propre au 11-Septembre que semble bel et bien s'inscrire le musée qui lui est consacré, la mise en scène des témoignages participant en effet à la réitération de l'événement.

Dans cette perspective, davantage encore que les médias traditionnels, ce sont les nouveaux médias qui occupent une place prépondérante dans le musée. Utilisés à de nombreuses reprises dans les deux principales expositions, ces outils tendent à personnaliser les attentats, en donnant la parole aux différents acteurs de l'événement, tout comme ils personnalisent le parcours de chaque visiteur. Au même titre que les artefacts amassés au fil des années et présentés dans les vitrines, le National September 11 Memorial Museum a constitué une vaste collection de témoignages oraux de témoins oculaires, de pompiers et secouristes, de survivants ou encore de proches des victimes. Le témoignage oral, sa collecte et sa préservation occupent une grande place dans le travail de documentation et de mémorialisation du 11-Septembre; de nombreuses initiatives ont fleuri en ce sens dès le lendemain des attentats, lancées par des institutions telles que la Bibliothèque du Congrès, l'université Columbia ou NPR (Kirshenblatt-Gimblett 27-30). En 2003, le producteur de radio David Isay imagine StoryCorps - un projet associatif à but non lucratif - et fait installer une cabine dans la gare Grand Central à Manhattan, dans laquelle les New-Yorkais sont invités à venir enregistrer des anecdotes personnelles. Constatant que de nombreuses personnes y enregistrent des hommages à des proches disparus le 11 septembre 2001 ou leurs souvenirs de cette journée, le National 9/11 Memorial Museum propose un partenariat à StoryCorps, donnant ainsi naissance à la September $11^{\text {th }}$ Initiative. Son principe est de recueillir au moins un témoignage de proche pour chaque victime des attentats de 1993 et de 2001. À ce jour, 
plus de mille témoignages ont été recueillis en mémoire de près de 600 victimes $^{12}$. Ces témoignages sont diffusés tout au long du parcours des visiteurs et notamment exploités dans deux installations : l'exposition In Memoriam et les soundscapes de l'exposition historique.

In Memoriam se présente comme un second mémorial à l'intérieur du musée, témoignant de la fonction commémorative de ces nouveaux médias. À l'intérieur d'une première galerie, les portraits de toutes les victimes ${ }^{13}$ des attentats du 11 septembre 2001 et de l'attentat de 1993 tapissent les quatre murs de la salle, du sol au plafond (figure 6).

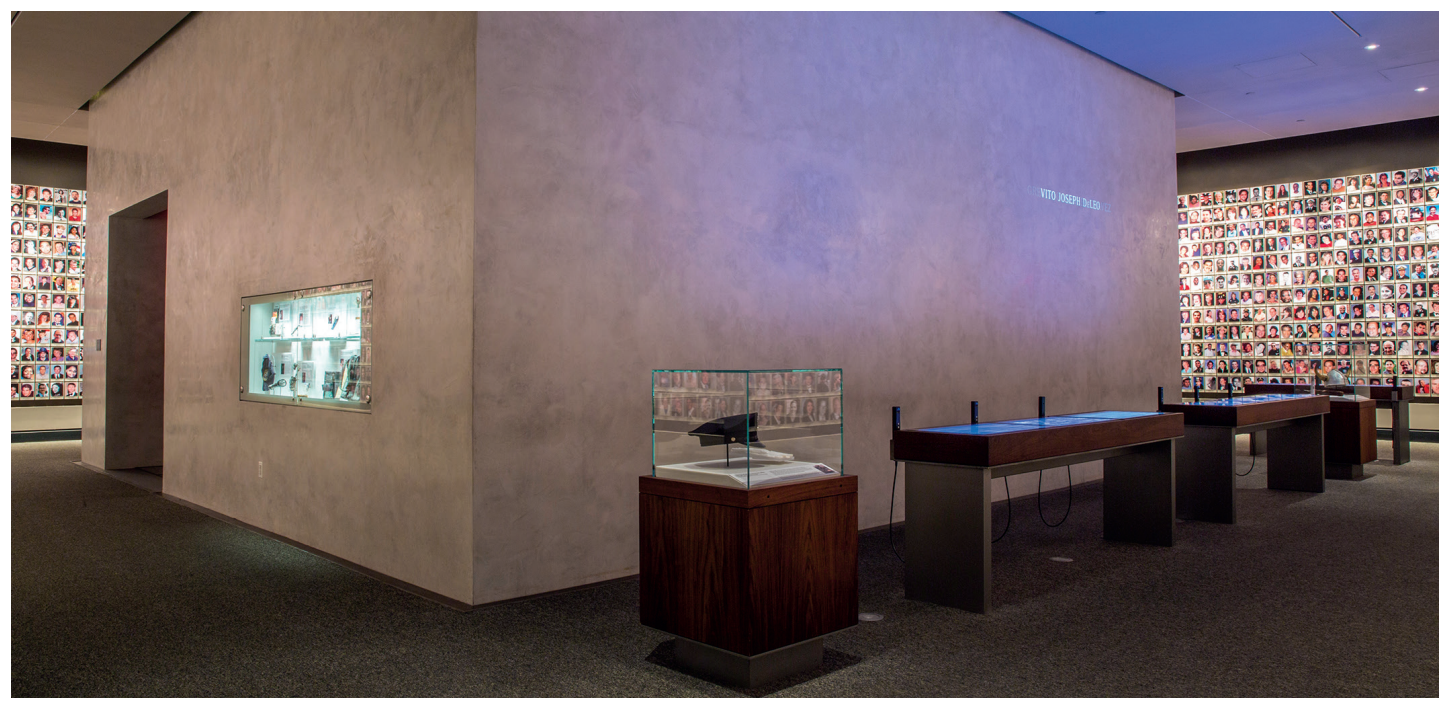

Figure 6 : Vue de l'exposition In Memoriam. On y aperçoit les portraits des victimes qui tapissent les murs et les tables avec écrans tactiles. Dans le cube blanc se trouve la salle dans laquelle sont projetés les portraits choisis par les visiteurs. Courtesy 9/11 Memorial Museum. Photographie de Jin S. Lee.

12. En 2011, à l'occasion du dixième anniversaire du 11 septembre 2001, StoryCorps réalise une série de courts métrages animés à partir de ces témoignages.

13. Cinq portraits de victimes ne figurent pas dans la galerie et sont remplacés par une feuille de chêne bicolore - l'espèce choisie pour le 9/11 Memorial. Pour trois d'entre elles cette absence relève d'une décision familiale ; quant aux deux autres victimes, le 9/11 Museum n'est toujours pas parvenu à obtenir leur portrait mais poursuit ses recherches en ce sens (9/11 Memorial Staff n.p. ; Dunlap et Beachy n.p.). 
Le long de la galerie sont installés des écrans tactiles grâce auxquels les visiteurs peuvent accéder à une petite biographie de chacune des victimes, enrichie de reproductions numériques d'objets personnels et d'un ou plusieurs témoignages oraux de leurs proches (figure 7). Depuis ces écrans, les visiteurs peuvent choisir de diffuser un de ces portraits dans une plus petite salle au centre de la galerie (figure 8).

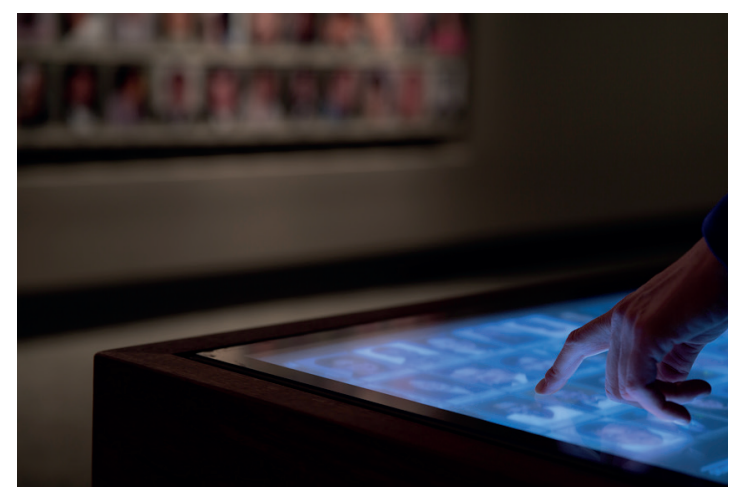

Figure 7 : Vue rapprochée d'une table avec écran tactile dans l'exposition In Memoriam. Courtesy 9/11 Memorial Museum. Photographie de Jin S. Lee.

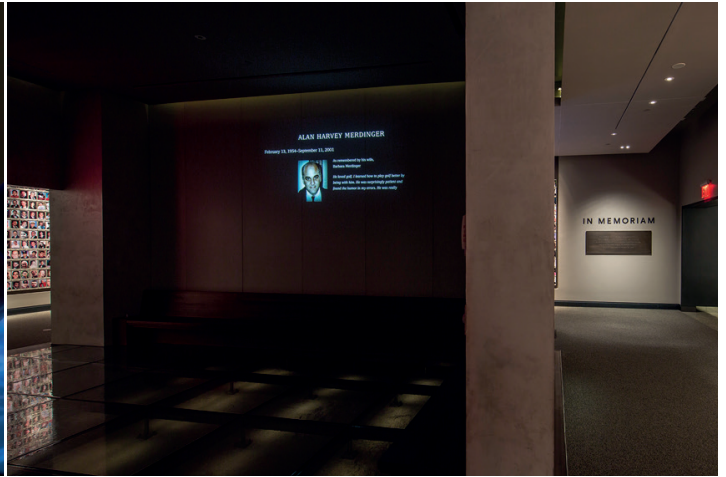

Figure 8 : Vue de l'exposition In Memoriam. On y aperçoit une partie de la galerie ainsi que l'intérieur de la salle dans laquelle sont projetés les portraits choisis par les visiteurs. Courtesy 9/11 Memorial Museum. Photographie de Jin S. Lee.

À la manière d'une petite chapelle, cette pièce sombre équipée de bancs invite au recueillement et à la contemplation de ces portraits, telles des reliques numériques. Dans l'exposition historique du musée, outre la diffusion de documents sonores d'archives - journaux d'information, derniers messages téléphoniques enregistrés par les passagers du vol 93-, plusieurs petits espaces, appelés soundscapes, diffusent les témoignages de survivants ou de témoins des attentats de New York simultanément à la projection d'un schéma indiquant leur emplacement par rapport aux tours jumelles au moment des attaques. Là encore, le dispositif audiovisuel prend place dans un petit espace sombre, sorte d'alcôve équipée de bancs ; en retrait du parcours, les soundscapes invitent le visiteur à interrompre sa déambulation et à s'asseoir quelques instants à l'écoute de souvenirs partagés, dans une sorte de tête-à-tête intime entre deux individus. 
Une des principales fonctions des nouveaux médias déployés dans le National September 11 Memorial Museum est donc, semble-t-il, de restituer une parole aux témoins, qui sont parfois euxmêmes des survivants des attentats. De même que les photographes amateurs avaient participé en masse à l'enregistrement de l'événement, le 11-Septembre nous est ainsi raconté, tout au long du parcours, à la première personne. En s'en remettant à cette parole grâce à la diffusion de témoignages vocaux ou d'archives sonores, le script de l'exposition semble soigneusement éviter toute analyse des événements et préférer alors un traitement plutôt affectif de son sujet. Si l'on peut, en effet, considérer que les enjeux et implications politiques des attentats, ainsi que la question de leur responsabilité n'ont pas leur place dans un mémorial dédié au recueillement et à la commémoration - qui plus est situé sur les lieux mêmes de la tragédie -, l'on ne peut cependant ignorer la prétention historique du National September 11 Memorial Museum. Les médias mis en œuvre dans le musée apparaissent alors clairement comme des outils appelant une réponse émotionnelle et compassionnelle, neutralisant tout positionnement critique.

L'hypertrophie du témoignage dans un tel contexte pose la question de la place du témoin dans la construction de l'histoire et l'élaboration d'une mémoire collective. Après avoir été reconnue en 1961, lors du procès Eichmann à Jérusalem ${ }^{14}$, c'est cependant aux États-Unis que la figure du témoin s'est d'abord imposée (Hartog 238) et c'est bien dans cette « ère du témoin », comme l'appelle Annette Wieviorka dans un ouvrage éponyme, que s'inscrit le musée du 11 septembre 2001. Selon François Hartog, le témoin - considéré comme le «porteur de mémoire » par excellence - s'est imposé dans notre espace public jusqu'à être aujourd'hui « reconnu, recherché, présent, voire à première vue omniprésent » (238). Cette figure est au centre d'une réflexion sur la dialectique entre mémoire et histoire, telle qu'elle est notamment déployée dans le National September 11 Memorial Museum. En effet, à travers le témoignage, mémoire et histoire sont souvent confondues, le témoignage étant alors considéré comme « un préalable à la connaissance des faits, base du futur récit historique » (Chevalier n.p.). Pour reprendre les mots d'Annette Wieviorka dans L'Ére du témoin, « le témoignage s'adresse au cœur, et non à la raison. Il suscite la compassion, la pitié, l'indignation, la révolte même parfois » (179). Pour Yves Chevalier, bien que ces sentiments soient tout à fait honorables, un historien ne peut que s'en distancer. Selon lui « la rigueur du récit historique ne peut être atteinte sous le signe et la pression des émotions » (n.p.). Toutefois, comme le souligne Amy Sodaro, plutôt que de recourir à des témoignages personnels pour illustrer ou compléter le récit historique, c'est sur la base de ceux-ci que s'écrit l'histoire dans le 9/11 Museum (143). Or c'est bien l'émotion et la compassion du visiteur que convoque la scénographie du National 9/11 Memorial Museum. Ces témoignages apparaissent alors

14. Lors du procès d'Adolf Eichman - haut fonctionnaire du Troisième Reich condamné pour crimes de guerre, crimes contre le peuple juif et crimes contre l'humanité notamment - de nombreux survivants ont été invités à témoigner pour raconter le génocide. Ce procès marque ainsi l'avènement du témoin dans l'écriture du récit historique. 
comme des outils curatoriaux permettant au musée de trouver sa voix/e, en évitant d'aborder autant que faire se peut, les questions d'ordre politique et géopolitique liées aux attentats.

En outre, ces archives numériques - enregistrements audio et artefacts numérisés - permettent de rendre plus personnelles et plus tangibles les pertes causées par les attentats de septembre 2001. Les individus disparus dans ces attaques émergent alors d'une masse de près de 3000 victimes, peut-être trop abstraite. Dans In Memoriam, le visiteur découvre des noms, des frères, des parents, des enfants, des gens qui aimaient lire, jouer aux tennis ou aller au cinéma, des gens comme lui, en somme. Aucune des victimes n'est laissée dans l'ombre ; chacune a droit à son nom gravé dans le mémorial ainsi qu'à sa photographie et son portrait numérique dans In Memoriam $^{15}$. Selon Erika Doss, les mémoriaux commémorant des attentats terroristes, récents dans l'histoire commémorative américaine, ont pris pour modèle les war memorials (monuments aux morts) du $\mathrm{XX}^{\text {ème }}$ siècle, et se sont ainsi construits sur le registre de l'héroïsation des victimes (159). Les morts, traités en héros, y sont célébrés individuellement et le nom de chacun est alors inscrit sur le monument. Cette tendance du naming, caractéristique des war memorials, est ainsi prégnante dans les pratiques commémoratives des attentats du 11 septembre 2001. Dès le lendemain des attaques, le New York Times commença ainsi à publier des petits portraits de chaque victime ; le recueil, intitulé Portraits of Grief, est aujourd'hui accessible sur le site web du journal et régulièrement mis à jour lors des anniversaires des attaques ${ }^{16}$. On a pu assister à une pratique similaire dans la presse française lors des attentats du 13 novembre 2015 contre le Bataclan et plusieurs terrasses dans les rues de Paris. Cette tendance est centrale dans le National 9/11 Memorial Museum, et dans In Memoriam en particulier. Les outils numériques permettent d'aller encore plus loin dans la personnalisation du mémorial et de dépasser la simple mention du nom. Identifier, nommer les victimes dans le musée souligne la barbarie des terroristes s'attaquant à des innocents et permet des allers-retours entre l'individu et la nation - s'attaquer à des individus « comme les autres », c'est viser la population dans son ensemble - et de dépolitiser les causes de l'événement - les victimes n'avaient rien fait pour mériter un tel sort. Erika Doss écrit ainsi que la pratique du naming construit un récit en deux temps : d'abord elle individualise, ensuite elle unifie ; d'abord elle présente les victimes comme des individus pleurés par leurs proches, puis comme un ensemble représentant le corps de la nation. Ainsi le naming permet-il de dépolitiser l'événement, tout en justifiant les décisions politiques prises pour y répondre

15. Le décompte des victimes des attentats du 11 septembre 2001 est cependant sujet à débat. En effet, un certain nombre de personnes sont décédées des suites de maladies ou traumatismes causés par les attentats, plusieurs mois, voire plusieurs années, après ceux-ci, mais ne sont pas comptabilisées dans les 2977 victimes.

16. Voir : http://www.nytimes.com/interactive/us/sept-11-reckoning/portraits-of-grief.html?8qa\&_r=0 (page consultée le 20 mai 2019). 
$(152)^{17}$. Donner à voir l'attentat depuis l'individu transforme l'événement en quelque chose d'imprévisible, gratuit et indépendant de tout contexte, et permet d'éviter la mise en cause du gouvernement américain.

À travers ces différents dispositifs multimédias, il semble finalement bel et bien que le visiteur soit à son tour convié à devenir ou redevenir lui-même témoin de ces attentats, avec la même émotion et la même stupeur. Il est intéressant de noter à ce propos le dispositif dans lequel sont présentées les photographies des «jumpers », telles que Falling Man de Richard Drew. En effet, bien que très célèbres et faisant partie des photographies iconiques du 11 septembre 2001, celles-ci sont exposées dans de petites alcôves, en retrait du parcours et à l'abri des regards les plus vulnérables, précédées d'une mise en garde à l'attention des visiteurs ; pour ceux qui osent se confronter à ces images, une boîte de mouchoirs en papier est à disposition. Plutôt qu'invité à réfléchir sur les attentats de septembre 2001, le spectateur semble invité à les revivre. En effet, au moyen des installations multimédias mises en place tout au long du parcours, c'est une véritable expérience immersive et interactive qui lui est proposée.

Dans une volonté encyclopédique indéniable, le musée met à la disposition du visiteur une très grande quantité d'informations dont il ne pourra prendre connaissance de façon exhaustive pendant sa visite. Le visiteur choisit ainsi celles qu'il souhaite consulter, si bien que chacun quitte le musée avec un contenu différent. Si le National 9/11 Memorial Museum regorge d'images fixes et vidéo, sa singularité tient encore davantage, comme on l'a montré, à l'omniprésence du son dans les expositions. En plus de favoriser l'immersion totale du visiteur dans le récit des attentats, le son apparaît comme le médium le plus à même de solliciter des réactions d'ordre affectif de la part du public. Plus que les photographies des victimes, les séquences-chocs du crash de l'avion dans la Tour Sud, ou encore les objets personnels calcinés, l'audio semble être le médium le plus efficace pour atteindre et engager le visiteur. Les messages vocaux des passagers du vol 93 appelant leurs proches pour leur dire au revoir, ou parfois les rassurer, comptent parmi les éléments les plus bouleversants du musée. Comme le suggère Marita Sturken, la diffusion de ces voix renforce la recherche d'authenticité dont le musée est empreint, le visiteur pouvant ainsi entendre l'émotion du locuteur dans son ton et son intonation («The 9/11 Memorial Museum » 485). Les voix des victimes fonctionnent de plus comme des reliques par excellence. La relique, et la question du sacré qui lui est associé, est omniprésente dans le musée, lui-même construit comme un véritable reliquaire ${ }^{18}$. L'exposition historique est en effet

17. Erika Doss écrit : « First individualizing and then unifying, first describing the dead as persons to be mourned and then claiming them as a national body, naming's two-step narrative depoliticizes (or evades) issues of responsibility and simultaneously justifies national imperatives of resolve and retribution » (152).

18. Le National 9/11 Memorial Museum renferme de véritables reliques puisqu'une de ses salles — accessible uniquement aux familles des victimes — contient des débris de corps encore non identifiés. 
constituée en grande partie de banals objets du quotidien (montres, portefeuilles, chaussures, etc.) qui acquièrent une valeur historique en intégrant les collections du musée (Sturken, « The objects that lived»19). De même que des reliques sacrées, de tels objets agissent, selon Krzysztof Pomian, «par la puissance évocatrice de [leur apparence] externe ou par la légende qui [leur] est attachée ou par les deux ensemble » (121) et leur exposition vise alors un sentiment d'identification de la part du public. Les voix apparaissant comme un lien fort et concret - malgré leur immatérialité - entre le visiteur et le témoin et ainsi, également, entre présent et passé. Lors de cette expérience immersive et interactive, véritable réitération de l'événement, le visiteur semble finalement devenir à son tour un témoin oculaire des attentats répétés - voire rejoués - inlassablement dans le musée, telle la diffusion sur un écran de télévision, en boucle et à l'infini, du crash du second avion dans la tour sud. Comme le souligne Lauren F. Klein, à travers l'ensemble des artefacts et dispositifs mis en scène, le musée tend à reproduire le trauma initial provoqué par les attentats (433).

Au moyen de ces nombreux outils numériques et installations multimédias, le National 9/11 Memorial Museum tente de jongler entre les multiples fonctions qui lui sont assignées. L'ensemble du site, constitué du mémorial en surface et du musée souterrain, s'appelle le National Septembre 11 Memorial and Museum, distinguant ainsi les deux fonctions du site, commémorative et muséale, en deux lieux différents. Cependant, le nom officiel du musée, National 9/11 Memorial Museum, stipule bien qu'il ne s'agit pas « seulement» d'un musée, mais également d'un mémorial. Or, musée et mémorial désignent des lieux dont certains aspects s'opposent. Si tous deux reposent sur quelque chose, d'une part, conservé, et d'autre part, rendu public, ils ne visent cependant pas les mêmes fins. Dans le cas du musée, conservation et diffusion ont pour but la construction et la transmission d'un savoir, qu'il soit artistique, scientifique ou encore historique par exemple ; le mémorial, quant à lui, tend à conserver le souvenir de quelqu'un ou de quelque chose jugée importante pour l'identité d'une communauté particulière, qu'elle soit locale, nationale ou éventuellement mondiale. Selon Dacia Viejo-Rose, les mémoriaux se tiennent alors dans l'interstice entre histoire et mémoire, entre affect et identité, et finalement entre passé, présent et futur (466). Or, c'est bien ces entre-deux que semble occuper le National 9/11 Memorial Museum.

Comme le souligne Erika Doss, les mémoriaux dédiés à des attentats terroristes ont des rôles bien distincts selon les différents groupes prenant part à leur élaboration. Pour les familles des victimes et les survivants, ce sont des lieux sacrés de deuil et souvent des sépultures. Pour les politiques, ils constituent des marqueurs idéologiques. Enfin, pour les touristes, ce sont des destinations de « tourisme 
noir $»^{19}$ (122). Il apparaît que le National 9/11 Memorial Museum peine à se positionner parmi les nombreuses fonctions qui sont les siennes, et que Marita Sturken énumère ainsi : « un mémorial aux victimes, un musée d'histoire, un tombeau, un lieu sacré, une destination touristique. Et également, une entreprise commerciale $»^{20}$ (« The 9/11 Memorial Museum »487). Situé sur les lieux mêmes de l'attaque qui fit le plus de victimes le 11 septembre 2001, et renfermant entre ses murs des débris de victimes encore non identifiés, il n’y a, littéralement, pas le recul nécessaire pour en faire un véritable musée d'histoire. Conçu tel un appendice pédagogique et expérimental du mémorial, le musée se déploie autour de thèmes tels que l'exceptionnalisme de ces attentats, l'héroïsme du peuple américain, ou encore la résilience de la nation, et la construction de son récit relève d'une véritable négociation entre les différents partis pris. La place à donner aux terroristes de ces attentats fut ainsi un des aspects les plus épineux du récit (Beard 155) et le traitement qui est fait de l'organisation terroriste islamiste Al-Qaïda dans l'exposition dite historique illustre bien le malaise du musée à proposer une analyse des attentats.

Dans son déploiement scénographique et notamment dans l'usage des outils multimédias, le National September 11 Memorial Museum semble donc ne pas assumer pleinement son statut de musée, voire de musée d'histoire. En reprenant la définition d'un mémorial donnée par Erika Doss, l'on pourrait dire que le National 9/11 Memorial Museum est un véritable « entrepôt de sentiments et d'émotions » (13). À la fois musée et mémorial, ce lieu à la forme hybride a la dure tâche de trouver un équilibre entre éducation et émotion, ainsi qu'entre écriture de l'histoire et préservation d'une mémoire. Situé sous les anciennes Twin Towers et sous l'actuel mémorial, le National 9/11 Memorial Museum a une influence prépondérante sur le récit historique et la mémoire culturelle des attentats de septembre 2001 (Sturken, "The Objects that lived »13). Imaginé dès 2006, mais achevé et inauguré il y a à peine cinq ans, le National September 11 Memorial Museum est un musée médiatique dans tous les sens du terme. Consacré à un événement historique et médiatique, organisé autour de nombreux médias numériques, il a fait couler beaucoup d'encre dans la presse à son ouverture. Le recours aux médias dans ses expositions apparaît alors comme un moyen de raconter l'événement sans aborder les questions encore brûlantes de la responsabilité de ces attentats, au risque cependant que l'émotion l'emporte sur l'éducation. Au-delà

19. Erika Doss écrit : «Terrorism memorials are the primary public sites where the nation remembers the victims of violent acts of extremism. For family members and survivors, they are sacred sites of bereavement and, ofter, burial. For politicians, they are ideological rallying grounds. For millions of tourists, they are "authentic" destinations marked by tragic death and traumatic loss » (122).

20. Je traduis : « a memorial to those who died, a historical museum, a shrine, a sacred site, a tourist destination. It is also a commercial venture. » Elle fait référence, en dernier lieu, à la boutique du musée, vendant notamment nombre de bibelots à l'effigie des Twin Towers ou autres symboles liés aux attentats — les pompiers ou les chiens de secours par exemple. 
du mardi 11 septembre 2001, le National 9/11 Memorial Museum apparait finalement comme un événement culturel symptomatique de la politique américaine post-11 septembre et un cas d'étude sans doute fructueux dans l'analyse de la construction du récit officiel de la « guerre contre la terreur ».

\section{Ouvrages CITÉS}

9/11 Memorial Staff. «Long-Sought Photos of 9/11 Victims Added to Memorial Portrait Wall ». The Memo Blog (3 janvier 2017). URL : https://www.911memorial.org/blog/long-sought-photos-911-victims-addedmemorial-portrait-wall (page consultée le 20 mai 2019).

BEARD, Rick. « Exhibit Review: The National September 11 Memorial \& Museum ». The Public Historian 37.1 (février 2015) : 150-158.

CHÉROUX, Clément. Diplopie. L'Image photographique à l'ère des médias globalisés. Essai sur le 11 septembre 2001. Paris : Le Point du Jour, 2009.

CHEVALIER, Yves. «Wieviorka (Annette). L'ère du témoin ». Archives de sciences sociales des religions 110 (avril-juin 2000). URL : http://assr.revues.org/20611 (page consultée le 20 mai 2019).

DOSS, Erika Lee. Memorial Mania: Public Feeling in America. Chicago : University of Chicago Press, 2010.

DUNLAP, David W. et Susan C. BEACHY. « Seeking the Final Faces for a 9/11 Tapestry of Grief, Loss, Life and Joy ». The New York Times (10 septembre 2016). URL : https://www.nytimes.com/2016/09/11/ nyregion/9-11-victims-portraits-national-september-11-memorial-museum.html (page consultée le 20 mai 2019).

FRAU-MEIGS, Divina. « Les clivages de l'américanéité, au miroir des médias ». Quaderni 50-51 (printemps 2003) : 155-173.

GERVAIS, Thierry. La Fabrique de l'information visuelle. Photographies et magazines d'actualité. Paris : Textuel, 2015.

GOODE, George Brown. «The relationships and responsibilities of museums ». 1895. Museum Origins: Readings in Early Museum History and Philosophy. Dir. Hugh H. Genoways et Mary Anne Andrei. Walnut Creek (CA) : Left Coast Press, 2008. 111-124.

HARTOG, François. Évidence de l'histoire. Ce que voient les historiens. 2005. Paris : Folio, 2007.

KIRSHENBLATT-GIMBLETT, Barbara. « Kodak moments, flashbulb memories. Reflections on 9/11». The Drama Review 47.1 (printemps 2003) : 11-48.

KLEIN, Lauren F. «Timescape and Memory. Visualizing Big Data at the 9/11 Memorial Museum ». The 
Routledge Companion to Media Studies and Digital Humanities. Dir. Jentery Sayers. New York : Routledge, 2018. 433-442.

NEWSEUM. Running toward Danger: Stories Behind the Breaking News of 9/11. New York : Rowman \& Littlefield Publishers, 2002.

PEARCE, Susan. « Studying museum material and collections ». International Journal of Heritage Studies 1.1 (1994) : 30-39.

POMIAN, Krzysztof. « Le musée face à l'histoire ». L'Histoire au musée. Actes du colloque. Dir. collectif. Arles : Actes Sud, 2004. 99-126.

SIRINELLI, Jean-François. «L'événement-monde. » Vingtième Siècle. Revue d'histoire 76 (2002/4) : 35-38.

SODARO, Amy. Exhibiting Atrocity. Memorial Museums and the Politics of Past Violence. New Brunswick (NJ) : Rutgers University Press, 2018.

STURKEN, Marita. «The 9/11 Memorial Museum and the remaking of Ground Zero ». American Quaterly 67.2 (juin 2015) : 471-490.

—. «The objects that lived: The 9/11 Museum and material transformation ». Memory Studies 9.1 (janvier 2016) : 13-26.

VIEJO-ROSE, Dacia. « Memorial functions. Intent, impact and the right to remember ». Memory Studies 4.4 (octobre 2011) : 465-480.

WIEVIORKA, Annette. L'Ère du témoin. 1998. Paris : Fayard, 2013.

Résumé : Le National September 11 Memorial Museum, à la fois musée et mémorial, a recours à de nombreux médias dans ses expositions. En explorant le rôle joué par ces outils numériques dans le récit des attentats du 11-Septembre fait par le musée, cet article suggère qu'ils permettent, d'une part, de raconter l'Histoire à la première personne en mettant en scène la figure du témoin et, d'autre part, de provoquer une réponse émotionnelle et compassionnelle chez le visiteur invité à une expérience interactive et immersive, brouillant ainsi les pistes entre les fonctions informatives et commémoratives de cette institution.

\begin{abstract}
The National September 11 Memorial Museum, both a museum and a memorial, uses several media in its exhibitions. Exploring the role played by these digital tools in the 9/11 narrative staged in the museum, this article considers, on the one hand, how they tell a first-person narrative through the figure of the witness, and, on the other hand, how they target an emotional reaction and awake empathy in the visitor who lives an immersive and interactive experience that blurs the lines between the informative and commemorative functions of this institution.
\end{abstract}

